\title{
Aripiprazole as a Candidate Treatment of COVID-19 Identified Through Genomic Analysis
}

\section{OPEN ACCESS}

Edited by:

Siddappa N Byrareddy, University of Nebraska Omaha, United States

Reviewed by: Sanjay Rathod, University of Pittsburgh, United States Subhash Chand, University of Nebraska Medical Center, United States

*Correspondence: Benedicto Crespo-Facorro benedicto.crespo.sspa@ juntadeandalucia.es

${ }^{t}$ These authors have contributed equally to this work

${ }^{\text {F}}$ These authors share senior authorship

Specialty section: This article was submitted to Inflammation Pharmacology,

a section of the journal

Frontiers in Pharmacology

Received: 27 December 2020 Accepted: 08 February 2021

Published: 02 March 2021

Citation:

Crespo-Facorro B, Ruiz-Veguilla M,

Vázquez-Bourgon $\mathrm{J}$,

Sánchez-Hidalgo $A C$

Garrido-Torres N, Cisneros JM,

Prieto C and Sainz J (2021)

Aripiprazole as a Candidate Treatment of COVID-19 Identified Through Genomic Analysis.

Front. Pharmacol. 12:646701. doi: 10.3389/fphar.2021.646701

\begin{abstract}
Benedicto Crespo-Facorro ${ }^{1,2 * t}$, Miguel Ruiz-Veguilla ${ }^{1,2+}$, Javier Vázquez-Bourgon ${ }^{2,3,4}$, Ana C. Sánchez-Hidalgo ${ }^{2,5}$, Nathalia Garrido-Torres ${ }^{1}$, Jose M. Cisneros ${ }^{6,7}$, Carlos Prieto ${ }^{8 \neq}$ and Jesus Sainz ${ }^{\text {9F }}$

${ }^{1}$ Department of Psychiatry, School of Medicine, University Hospital Virgen del Rocio-IBIS, Sevilla, Spain, ${ }^{2}$ Spanish Network for Research in Mental Health (CIBERSAM), Sevilla, Spain, ${ }^{3}$ Department of Psychiatry, University Hospital Marques de Valdecilla Instituto de Investigacion Marques de Valdecilla (IDIVAL), Santander, Spain, ${ }^{4}$ Department of Medicine and Psychiatry, School of Medicine, University of Cantabria, Santander, Spain, ${ }^{5}$ Seville Biomedical Research Centre (IBiS), Sevilla, Spain, ${ }^{6}$ Department of Infectious Diseases, Microbiology and Preventive Medicine, Institute of Biomedicine of Seville, University Hospital Virgen del Rocio, University of Seville, Salamanca, Spain, ${ }^{7}$ Spanish Network for Research in Infectious Diseases (REIPI), Madrid, Spain, ${ }^{8}$ Bioinformatics Service, Nucleus, University of Salamanca, Salamanca, Spain, ${ }^{9}$ Spanish National Research Council (CSIC), Institute of Biomedicine and Biotechnology of Cantabria, Santander, Spain
\end{abstract}

Background: Antipsychotics modulate expression of inflammatory cytokines and inducible inflammatory enzymes. Elopiprazole (a phenylpiperazine antipsychotic drug in phase 1) has been characterized as a therapeutic drug to treat SARS-CoV-2 infection in a repurposing study. We aim to investigate the potential effects of aripiprazole (an FDA approved phenylpiperazine) on COVID-19-related immunological parameters.

Methods: Differential gene expression profiles of non-COVID-19 vs. COVID-19 RNA-Seq samples (CRA002390 project in GSA database) and drug-naïve patients with non-affective psychosis at baseline and after three months of aripiprazole treatment were identified. An integrative transcriptomic analyses of aripiprazole effects on differentially expressed genes in COVID-19 patients was performed.

Findings: 82 out the 377 genes (21.7\%) with expression significantly altered by aripiprazole have also their expression altered in COVID-19 patients and in 93.9\% of these genes their expression is reverted by aripiprazole. The number of common genes with expression altered in both analyses is significantly higher than expected (Fisher's Exact Test, two tail; $p$ value $=3.2 \mathrm{e}-11) .11 \mathrm{KEGG}$ pathways were significantly enriched with genes with altered expression both in COVID-19 patients and aripiprazole medicated nonaffective psychosis patients ( $p$ adj $<0.05$ ). The most significant pathways were associated to immune responses and mechanisms of hyperinflammation-driven pathology (i.e.,"inflammatory bowel disease (IBD)" (the most significant pathway with a $p$ adj of $0.00021)$, "Th1 and Th2 cell differentiation" and "B cell receptor signaling pathway") that have been also associated with COVID19 clinical outcome.

Interpretation: This exploratory investigation may provide further support to the notion that a protective effect is exerted by aripiprazole (phenylpiperazine) by modulating the expression of genes that have shown to be altered in COVID-19 patients. Along with many 
ongoing studies and clinical trials, repurposing available medications could be of use in countering SARS-CoV-2 infection, but require further studies and trials.

Keywords: psychosis, inflammation, immunology, coronavirus, repurposing drugs, elopiprazole, SARS-CoV-2

\section{INTRODUCTION}

The SARS-CoV-2 epidemic has become the greatest challenge facing medicine today. Infected patients present with a wide range of clinical severity varying from asymptomatic to fatal condition (Wu et al., 2020). Advanced age, gender (male) and suffering comorbidities (diabetes, cardiovascular or chronic respiratory diseases) are risk factors for higher clinical severity, hospitalization rate and death from COVID-19 (Rubino et al., 2020; Zhou et al., 2020). The presence of these comorbidities may decrease resilience and lower the ability to tolerate additional cytokine storm (Mangalmurti and Hunter, 2020).

COVID-19 individuals who become critically and fatally ill seem to experience an indiscriminate and runaway immune response with an unchecked systemic overproduction of cytokines and immunological disbalance (Bhaskar et al., 2020; Manjili et al., 2020; Zeng et al., 2020). COVID-19 related immunopathogenesis is not understood just as an emergent cytokine storm but also as an impairment of protective $\mathrm{T}$ cell immunity (Chen et al., 2020).

There are no FDA-approved antivirals or vaccines for any coronavirus, including SARS-CoV-2, and current treatments for COVID-19 are limited to supportive therapies and off-label use of FDA-approved drugs. Anti-inflammatory drugs such as dexamethasone have been shown to reduce deaths (Vabret et al., 2020). The crucial role of NLRP3 inflammasome activation in the pathogenesis of diseases caused by SARSCoVs draws also attention toward potential role of its inhibitors in the treatment of COVID-19 (van den Berg and Te Velde, 2020). Wide range of different drug classes, such as cancer therapeutics, antipsychotics, and antimalarials, seem to have a beneficial effect against MERS and SARS coronaviruses (Dyall et al., 2017). Weston et al., (2020) observed that, although infection cannot be prevented, chlorpromazine (typical antipsychotic drug) and chloroquine protect mice from severe clinical disease from SARS-CoV. Clozapine (atypical antipsychotic drug) has revealed to be effective in suppressing the proinflammatory cytokine expression by limiting the NLRP3 inflammasome activation in vitro (Giridharan et al., 2020). In the same line as above, Riva and colleagues (2020) analyzed approximately 12,000 drugs in clinical-stage or Food and Drug Administration (FDA)approved small molecules to identify candidate drugs to treat COVID-19 and reported that elopiprazole (a never marketed phenylpiperazine antipsychotic drug) was listed among the 21 most potent compounds to inhibit SARS-CoV infection. Phenyl-piperazine derivatives had proved their utility as an effective source of antiviral compounds, with different mechanisms of action, for treatment of human adenovirus and cytomegalovirus (DNA viruses) (Sanchez-Cespedes et al., 2016).
Antipsychotics suppress expression of inflammatory cytokines and inducible inflammatory enzymes (i.e., cyclooxygenase) and microglia activation (Dinesh et al., 2020). These antiinflammatory effects are elicited via the reduction of proinflammatory cytokines production, modulating monocytes response through TLR and the inhibition of the microglial activation by reducing the levels of inducible nitric oxide synthase (iNOS), IL-1 $\beta$, IL-6, and TNF- $\alpha$ (Kato et al., 2007; Obuchowicz et al., 2017). In humans, the immunomodulatory effect of risperidone (pyridopyrimidines) and aripiprazole (marketed phenylpiperazine) has been demonstrated (JuncalRuiz et al., 2018), with aripiprazole demonstrating a greater anti-inflammatory effect on TNF- $\alpha$, IL-13, IL-17 $\alpha$ and fractalkine. Thus, the protective effect of phenylpiperazine marketed antipsychotics (aripiprazole) against a pernicious cytokine storm is a hypothesis that warrants further investigation with the aim of unrevealing new off-label drug to be use in severe COVID19 patients.

Prevalence and severity of COVID-19 infection in patients with severe mental disorders have yielded to inconsistent results, likely due to differences in the methodology utilized in these investigations. Lee and collaborators (2020) reported that diagnosis of a mental disorder was not associated with increased likelihood of SARS-CoV infection, but a slightly higher risk for severe clinical outcomes (Lee et al., 2020). Wang and colleagues (2021) reported a higher overall risk to get infected among schizophrenia patients. In a retrospective epidemiological study, we observed that vulnerable severe mental disorder individuals on long-acting injectable antipsychotics had a lower risk of SARS-CoV2 infection and a better outcome after infection (unpublished data).

The aim of the present study was to examine the potential beneficial effects of aripiprazole (antipsychotic) in COVID-19 infection by: 1.- analyzing the profile of gene expression of drugnaïve patients with psychosis at baseline and after three months of treatment with aripiprazole (PAFIP sample); and 2.- comparing the set of genes with altered expression in COVID-19 patients (Wuhan sample) with the set of genes modulated by aripiprazole in drug-naïve schizophrenia patients.

\section{MATERIALS AND METHODS}

\section{PAFIP Cohort of Aripiprazole-Treated Patients \\ Setting and Sample Study}

The cohort analyzed to study the effect of aripiprazole was obtained at the University Hospital Marques de Valdecilla (Cantabria, Spain). Conforming to international standards for research ethics, this study was approved by the Cantabria Ethics Institutional Review Board (IRB). Patients meeting 
inclusion criteria for a first episode of non-affective psychosis (drug-naïve) provided written informed consent to be included in the study. After informed consent was signed, patients were included in a prospective, randomized, flexibledose, open-label study (Crespo-Facorro et al., 2017; Mayoral Van-Son et al., 2021).

\section{Laboratory Assessments}

Blood samples were obtained from 57 fasting non-affective psychosis subjects (25 males and 32 females; mean age of 31.54 years) from 8:00 to 10:00 a.m. by the same staff and in the same setting. A detailed description of methodology followed to assess biochemical variables is available upon request to the authors. None of the patients had a chronic inflammation or infection, or were taking medication that could influence the results of blood tests.

\section{RNA Extraction}

Total RNA was extracted from blood using the Tempus ${ }^{\mathrm{TM}}$ Blood RNA Tube and the Tempus ${ }^{\mathrm{TM}}$ Spin RNA Isolation Kit (Applied Biosystems, Foster City, CA, United States) following the manufacturer's protocols. To select only high-quality RNA, the RNA integrity number (RIN) was characterized with a Bioanalyzer (Agilent Technologies, Santa Clara, CA, United States) and samples with a RIN of at least 7.6 were used.

\section{RNA Next-Generation Sequencing}

Total RNA was extracted from peripheral blood of each individual. The messenger RNA (mRNA) obtained from blood was sequenced at the Centro Nacional de Análisis Genómico (CNAG) using Illumina HiSeq instruments (San Diego, CA, United States). The mRNA was isolated from the total RNA and was fragmented once transformed into complementary DNA (cDNA). Fragments of $300 \mathrm{bp}$ on average were selected to construct the cDNA libraries for sequencing. Pair-end sequences of 70 nucleotides for each end were produced. The mRNA from blood samples of 57 drug-naïve non-affective psychosis patients at baseline and after 3 months of continuous treatment with aripiprazole was sequenced.

Sequence files were aligned to the GRCh38 human reference genome (Gencode release 25) using the STAR aligner (Harrow et al., 2012; Dobin et al., 2013). Reads count were normalized with the Voom algorithm using the cyclic loess method (Law et al., 2014) and significant gene expression changes between treated and naïve patients were identified with lima (Smyth et al., 2005). We performed a paired analysis using a Wald test and a parametric fit type with an adjusted $p$-value cutoff of 0.01 .

\section{Wuhan COVID19 Dataset}

COVID data was downloaded from the GSA server (Wang et al., 2017) with the CRA002390 identifier (Xiong et al., 2020). This research collected RNA-Seq samples of peripheral blood mononuclear cells (PBMC) from three COVID-19 patients (all were males; mean age of 45.3 years) and three healthy donors at Zhongnan Hospital of Wuhan University that were included in the present study. These data were

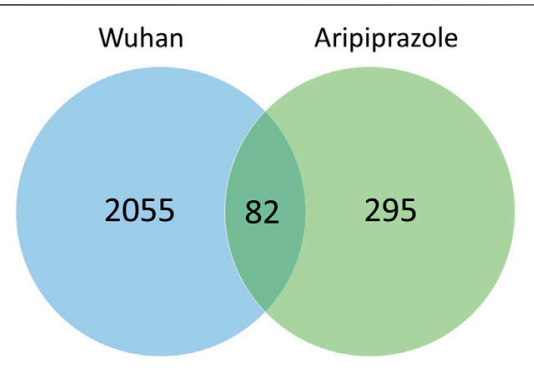

FIGURE 1 | Number of genes with expression altered in Wuhan sample (COVID-19) and PAFIP sample (aripiprazole-treated).

analyzed with RaNA-Seq (Prieto and Barrios, 2020) cloud platform and differential expression genes were detected by means of DESeq2 (Love et al., 2014) cloud using a Wald test, a parametric fit type and setting an adjusted $p$-value cutoff of 0.01 .

\section{Statistical and Bioinformatic Analysis}

Statistical significance of differential expression co-occurrence between COVID-19 and aripiprazole studies was calculated with a Fisher Exact test. Functional enrichment analyses, to identify biological pathways in the Biosystem database (downloaded on January 2020) (Geer et al., 2010) with a significant presence of differential expressed genes, were carried out with a Fisher Exact test. KEGG was used for representation and analysis of molecular networks (Kanehisa et al., 2010). Visualization and final representation of pathways were performed with the pathview R package (Luo and Brouwer, 2013).

\section{RESULTS}

Integrative transcriptomic analyses of aripiprazole effects on differentially expressed genes in COVID-19 patients:

\section{COVID-19 Patients Versus Healthy Donors (Wuhan Sample)}

We found 2,137 genes with significant differential expression between COVID-19 patients and controls ( $p$ adj value $<0.05$ ) (Supplementary Table S1). The most significant gene was the Charcot-Leyden crystal galectin gene (CLC) with a $p$ adj value of 7.8e-23.

\section{Drug-Naïve Non-affective Psychosis Patients at Entry and After 3 months of Aripiprazole Treatment (PAFIP Sample)}

We found 377 genes with significant differential expression before and after medication ( $p$ adj value $<0.05$ ) (Supplementary Table S2). The two most significant genes were the LIM domain only 4 (LMO4) and the ATP binding cassette subfamily A member 9 (ABCA9) with a $p$ adj value of 0.0039 . 
TABLE 1 | KEGG pathways significantly enriched with genes with altered expression in COVID19 patients and schizophrenia patients treated with aripiprazole.

\begin{tabular}{|c|c|c|c|c|c|c|c|}
\hline Pathway ID & Source & Pathway name & Observed \% & Expected \% & $p$ value Fisher & $\begin{array}{l}\text { No. of genes per } \\
\text { pathway }\end{array}$ & Gene symbol \\
\hline hsa05321 & KEGG & $\begin{array}{l}\text { Inflammatory bowel } \\
\text { disease (IBD) }\end{array}$ & 8.16 & 0.57 & 0.00021 & 4 & GATA3 HLA-DQB1 IL18 STAT4 \\
\hline hsa04658 & KEGG & Th1 and Th2 cell differentiation & 8.16 & 0.82 & 0.00079 & 4 & $\begin{array}{l}\text { GATA3 HLA-DQB1 NFATC2 } \\
\text { STAT4 }\end{array}$ \\
\hline hsa04380 & KEGG & Osteoclast differentiation & 8.16 & 1.17 & 0.00281 & 4 & BTK NFATC2 SYK LILRB4 \\
\hline hsa04664 & KEGG & Fc epsilon RI signaling pathway & 6.12 & 0.62 & 0.00382 & 3 & BTK PLA2G4A SYK \\
\hline hsa04662 & KEGG & B cell receptor signaling pathway & 6.12 & 0.65 & 0.00430 & 3 & BTK NFATC2 SYK \\
\hline hsa05164 & KEGG & Influenza A & 8.16 & 1.50 & 0.00653 & 4 & $\begin{array}{l}\text { HLA-DQB1 HLA-DQB1 IL18 } \\
\text { NLRP3 }\end{array}$ \\
\hline hsa04064 & KEGG & NF-kappa B signaling pathway & 6.12 & 0.84 & 0.00867 & 3 & BTK LTA SYK \\
\hline hsa04659 & KEGG & Th17 cell differentiation & 6.12 & 0.95 & 0.01202 & 3 & GATA3 HLA-DQB1 NFATC2 \\
\hline hsa04611 & KEGG & Platelet activation & 6.12 & 1.09 & 0.01712 & 3 & BTK PLA2G4A SYK \\
\hline hsa05166 & KEGG & HTLV-I infection & 8.16 & 2.33 & 0.02787 & 4 & FZD2 HLA-DQB1 LTA NFATC2 \\
\hline hsa05152 & KEGG & Tuberculosis & 6.12 & 1.53 & 0.03986 & 3 & HLA-DQB1 IL18 SYK \\
\hline
\end{tabular}

\section{Differential Gene Expression Between COVID-19 and Aripiprazole-Treated Samples}

82 out the 377 genes $(21.7 \%)$ modified by aripiprazole treatment (PAFIP sample) are significantly also altered in COVID-19 patients (Wuhan sample) (Figure 1). The number of common genes to both analyses is significantly higher than expected by chance (Fisher's Exact Test, two tail; $p$ value $=3.2 \mathrm{e}-11$ )

Interestingly, out of the 82 genes with expression altered in both analyzed cohorts 55 genes have decreased expression after aripiprazole medication and increased expression in COVID-19 patients; also, out of the 82 genes common to both cohorts 22 have increased expression after aripiprazole medication and decreased expression in COVID-19 patients. In total 77 genes out of 82 (93.9\%) have altered expression in different direction when we compared the effects of COVID-19 and aripiprazole medication.

\section{KEGG Pathways Significantly Enriched With Common Genes With Altered Expression in COVID-19 and Aripiprazole-Treated \\ Samples}

The analysis of pathways for enrichment of common genes with altered expression in COVID-19 and aripiprazole patients shows 11 pathways significantly enriched ( $p$ value Fisher <0.05) (Table 1). Several of those pathways are related to the immune system such as the "inflammatory bowel disease (IBD)" (the most significant pathway; $p$ adj of 0.00021 ), "Th1 and Th2 cell differentiation" and " $\mathrm{B}$ cell receptor signaling pathway," both related to the defense against infections.

\section{DISCUSSION}

Our results herein revealed that the differently expressed genes in COVID-19 patients and schizophrenia patients treated with aripiprazole were highly associated with numerous immune- related pathways, including "Inflammatory bowel disease (IBD)", "Th1 and Th2 cell differentiation", "Fc epsilon RI signaling pathway", "B cell receptor signaling pathway", "NFkappa B signaling pathway" and "Th17 cell differentiation". It is worth to remark that these immunological pathways have been associated with COVID19 clinical outcome. Thus, a wide array of host humoral and cellular immune response alterations associated with SARS-CoV-2 infection might cause an uncontrolled or insufficient immune response that may lead to immunopathology and cause severe damage to patients. (Tay et al., 2020; Wang et al., 2021). Lymphopenia marked by T cell and NK cell dysfunction, increases in proinflammatory markers and cytokines, and potential blood hypercoagulability characterize severe COVID-19 cases (Vabret et al., 2020).

In more severe COVID-19 cases, death results from hypoxemic respiratory failure in patients developing severe acute respiratory distress syndrome and is associated, in a substantial portion of patients, with an inflammatory syndrome and cytokine storm (Mehta et al., 2020) that may originate from immune cells (Chen et al., 2020). Longitudinal analysis of the immune response observed in a fatal case of COVID-19 revealed waves of a pro-inflammatory cytokine storm, Th1 and Th2 activation, and markers of $\mathrm{T}$ cell exhaustion, apoptosis, cell cytotoxicity, and endothelial activation were until the fatal outcome (Bouadma et al., 2020).

The TH17 type response profoundly also contributes to the cytokine storm in pulmonary viral infection including SARSCoV-2 (Josset et al., 2013). Compared with non-ICU COVID-19 patients, ICU COVID-19 patients have even higher levels of several cytokines specifically involved in TH17 type responses (Huang C. et al., 2020). It has been recently proposed that JAK2 inhibitor (Fedratinib@) can prevent the deteriorating outcomes of TH17 associated cytokine storm in COVID-19 by suppressing the production of several TH17 signature cytokines (Wu and Yang, 2020). Targeting the TH17 pathway may benefit the patients with TH17 dominant immune profiles.

Among biologic agents in patients with severe COVID-19 inhibiting Fc epsilon RI signaling has been proposed (Yalcin and Yalcin, 2021). Omalizumab specifically binds to the $\mathrm{CH} 3$ domain, is 
near to the binding site for the high affinity IgE Fc receptors type-I (also called FceRI) of human IgE (Metz et al., 2017). A critical issue in patients with Covid-19 is the viremia and the overresponse to this viremia with increase of ferritin, CRP and D-Dimer that are directly associated with the mortality (Huang I. et al., 2020). It has been described that omalizumab safely decreases the coagulant proteins (D-Dimer) and proinflammatory cytokines/mediators and increases the anti-coagulant proteins (protein C, S) in patients with sepsis (Yalcin et al., 2013; Criado et al., 2020). So, it could be anticipated that we may administer it for severe COVID-19 (Yalcin and Yalcin, 2021).

We observed that those genes differentially expressed in COVID-19 and aripiprazole-treated patients were involved in Inflammatory bowel disease (IBD). Autoinflammatory diseases (IBD) that present with bowel inflammation and intractable diarrhea owing to an inappropriate inflammatory response, with also altered key immune pathways underlying persistent inflammation such as excessive IL-1 signaling, constitutive NF- $\kappa \mathrm{B}$ activation, and chronic type I IFN signaling (de Jesus et al., 2015). It is also of interest that JAK-STAT pathways, as well as in COVID19, play an important role in the inflammatory response characteristic of IBD and represent a promising therapeutic target for treatment of the disease. (Pedersen et al., 2014).

In our study, differentially expressed genes involved in NFkappa B signaling pathway were identified according to KEGG pathway analysis. The activated NF- $\kappa B$ transcription factors serve as a "rapid acting" primary transcription factor regulating diverse cellular responses associated with chronic inflammatory states, septic shock syndrome and multiorgan failure (Zhang et al., 2017). Hyper-activation of the nuclear factor kappa-light-chain enhancer of activated B cells (NF-kB) pathway has been implicated in the pathogenesis of the severe/critical COVID19 phenotype (Hirano and Murakami, 2020). Cromolyn, which inhibit NF-kB mediated cytokine production, has been suggested as a repurposing drug in the fight against COVID19 (Karadsheh Adli, 2020). Many of the drugs currently effective in COVID disease appear to have links to the NF- $\mathrm{BB}$ cascade of immune regulation (Hariharan et al., 2020).

Despite all recent generated scientific knowledge, immune responses and mechanisms of hyperinflammation-driven pathology need to be further elucidated to address how these immune differences across patients or between different types of coronavirus infections dictate who succumbs to disease and who remains asymptomatic (Vabret et al., 2020).

The fact that antipsychotics have a demonstrated effect on immunological pathways, inducible inflammatory enzymes (i.e., cyclooxygenase), and microglia activation (Dinesh et al., 2020) may lead to the speculation about the beneficial effects of these drugs on controlling the acute hyper-inflammatory response that may be responsible for critical COVID19 illness. A recent study (Riva et al., 2020) profiled a library of approximately 12,000 drugs in clinical-stage or FDA-approved small molecules to identify candidate drugs to treat COVID-19. In the list of the 21 most potent compounds to inhibit infection validated in dose response across multiple cell lines there are two antipsychotic drugs, elopiprazole and 8-(3-Chlorostyryl) caffeine which are in phase I and preclinical stages respectively. Elopiprazole and aripiprazole belong to the class of organic compounds known as

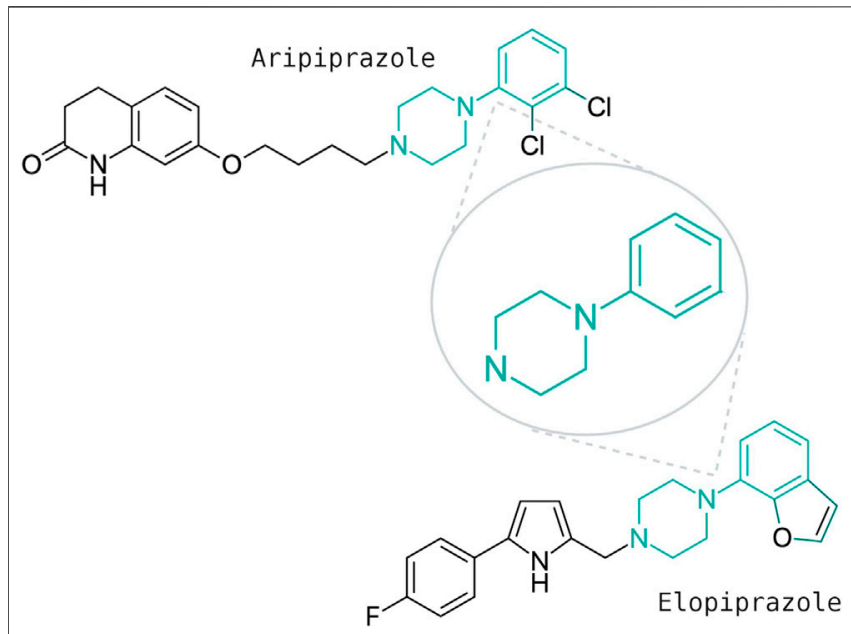

FIGURE 2 | Chemical structures of two phenypiperazines with antipsychotic effects: elopiprazole and aripiprazole.

phenylpiperazines, containing a phenylpiperazine skeleton, which consists of a piperazine bound to a phenyl group (Figure 2).

If our results herein prove to be certain one may expect a milder severity of COVID-19 in patients on aripiprazole (phenylpiperazine). Differences in the definitions and methodology utilized in recent investigations have yielded to inconsistent results about the prevalence and severity of COVID19 infection among patients with severe mental disorders (Lee et al., 2020; Wang et al., 2021). It is of worth to pinpoint that Wang and colleagues (2021) reported that chronic schizophrenia patients have a lower association compared with recent diagnosis of schizophrenia (less than 1 year $)(\mathrm{AOR}=1.48,95 \% \mathrm{CI}: 1.33-1.65$ vs. $\mathrm{AOR}=9.89$, 95\% CI: 8.68-11.26), giving place to the speculation that antipsychotic treatment may exert a protective effect. Nemani et al., (2021) recently described that schizophrenia spectrum diagnosis was associated with an increase of death or discharge to hospice outcome at 45 days following testing and highlighted the relevance of evaluating the potential protective effects of psychotropic medications. Our data retrospectively exploring an epidemiological sample of people with severe mental disorders who are on long-acting injectable antipsychotics revealed a lower risk of SARS-CoV2 infection and a better outcome after infection in this group of antipsychotic treated individuals compared to overall COVID-19 people (Canal-Rivero et al., 2021).

Some limitations need to be taken into when interpreting our results. First, differences in gender distribution between cohorts may limit the comparability of gene expression data. Second, the pattern of gene expression associated to COVID19 infection raises from three COVID19 patients and three healthy donors from whom there is limited access to subjects' characteristics.

\section{CONCLUSION}

Given that the effect of aripiprazole in gene expression mainly revert the changes in expression caused by COVID19 infection, 
and that another never marked phenylpiperazine antipsychotic (elopiprazole) has been validated as potential treatment for COVID19, it may be suggested that aripiprazole might be used as treatment for COVID19. Along with many ongoing studies and clinical trials, repurposing available medications could be of use in countering SARS-CoV-2 infection, but clearly require further studies and trials.

\section{DATA AVAILABILITY STATEMENT}

The datasets presented in this study can be found in online repositories. The names of the repository/repositories and accession number(s) can be found below: https://www.ebi.ac. uk/ena, PRJEB42627.

\section{ETHICS STATEMENT}

The studies involving human participants were reviewed and approved by the Cantabria Ethics Institutional Review Board (IRB). The patients/participants provided their written informed consent to participate in this study.

\section{AUTHOR CONTRIBUTIONS}

Study concept and design: BC-F, MR-V, CP, and JS; acquisition, analysis or interpretation of data: all authors; drafting of the manuscript: all authors; critical revision of the manuscript for important intellectual content: all authors; obtained funding: BC-F and JS; study supervision: BC-F, MR-V, CP, and JS.

\section{REFERENCES}

Bhaskar, S., Sinha, A., Banach, M., Mittoo, S., Weissert, R., Kass, J. S., et al. (2020). Cytokine storm in COVID-19-Immunopathological mechanisms, clinical considerations, and therapeutic approaches: the REPROGRAM consortium position paper. Front. Immunol. 11, 1648. doi:10.3389/fimmu.2020.01648

Bouadma, L., Wiedemann, A., Patrier, J., Surénaud, M., Wicky, P. H., Foucat, E., et al. (2020). Immune alterations in a patient with SARS-CoV-2-related acute respiratory distress syndrome. J. Clin. Immunol. 40 (8), 1082-1092. doi:10. 1007/s10875-020-00839-x

Canal-Rivero, M., Barragán, R. C., García, A. R., Garrido-Torres, N., CrespoFacorro, B., and Ruiz-Veguilla, M. (2021). Lower risk of SARS-CoV2 infection in individuals with severe mental disorders on antipsychotic treatment: A retrospective epidemiological study in a representative Spanish population. Schizophrenia Res. 229, 53-54. doi:10.1016/j.schres.2021.02.002

Chen, G., Wu, D., Guo, W., Cao, Y., Huang, D., Wang, H., et al. (2020). Clinical and immunological features of severe and moderate coronavirus disease 2019. J. Clin. Invest. 130 (5), 2620-2629. doi:10.1172/JCI137244

Crespo-Facorro, B., Ortiz-Garcia de la Foz, V., Suarez-Pinilla, P., Valdizan, E. M., Pérez-Iglesias, R., Amado-Señaris, J. A., et al. (2017). Effects of aripiprazole, quetiapine and ziprasidone on plasma prolactin levels in individuals with first episode nonaffective psychosis: analysis of a randomized open-label 1year study. Schizophr. Res. 189, 134-141. doi:10.1016/j.schres.2017.01.046

\section{FUNDING}

The present study was part of a larger prospective longitudinal study, the "First Episode Psychosis Clinical Program 10" (PAFIP10) study. ClinicalTrials.gov Identifiers: NCT02200588, NCT03481465, and NCT03476473. No pharmaceutical industry or institutional sponsors participated in the study conception and design, data collection, analysis and interpretation of the results, or drafting of the manuscript. This work was supported by: SAF201676046-R and SAF2013-46292-R (MINECO and FEDER) to B.C.F.

\section{ACKNOWLEDGMENTS}

We are highly indebted to the participants and their families for their cooperation in this study. We also thank IDIVAL biobank (Inés Santiuste and Jana Arozamena) for clinical samples and data as well as the PAFIP members (Marga Corredera) for the data collection. We kindly thank all clinical staff at the Hospital Universitario Virgen del Rocio for support to collect clinical records and provide clinical care to COVID-19 patients. We also kindly thank Dra. Marisa Barrigon for helpful discussions regarding clinical data analysis, and Idalino Rocha for manuscript editing and formatting. This manuscript has been released as a pre-print at medRxiv. Available at: https:/doi.org/ 10.1101/2020.12.05.20244590 (Crespo-Facorro et al., 2020).

\section{SUPPLEMENTARY MATERIAL}

The Supplementary Material for this article can be found online at: https:/www.frontiersin.org/articles/10.3389/fphar.2021.646701/ full\#supplementary-material.

Crespo-Facorro, B., Ruiz-Veguilla, M., Vázquez-Bourgon, J., Sánchez-Hidalgo, A. C., Garrido-Torres, N., Cisneros, J. M., et al. (2020). Aripiprazole as a candidate treatment of COVID-19 identified through genomic analysis. MedRxiv. doi:10. 1101/2020.12.05.20244590

Criado, P. R., Pagliari, C., Criado, R. F. J., Marques, G. F., and Belda, W. (2020). What the physicians should know about mast cells, dendritic cells, urticaria, and omalizumab during COVID-19 or asymptomatic infections due to SARS-CoV2? Dermatol. Ther. 33 (6), e14068. doi:10.1111/dth.14068

de Jesus, A. A., Canna, S. W., Liu, Y., and Goldbach-Mansky, R. (2015). Molecular mechanisms in genetically defined autoinflammatory diseases: disorders of amplified danger signaling. Annu. Rev. Immunol. 33, 823-874. doi:10.1146/ annurev-immunol-032414-112227

Dinesh, A. A., Islam, J., Khan, J., Turkheimer, F., and Vernon, A. C. (2020). Effects of antipsychotic drugs: cross talk between the nervous and innate immune system. CNS Drugs 34, 1229. doi:10.1007/s40263-020-00765-x

Dobin, A., Davis, C. A., Schlesinger, F., Drenkow, J., Zaleski, C., Jha, S., et al. (2013). STAR: ultrafast universal RNA-seq aligner. Bioinformatics 29 (1), 15-21. doi:10. 1093/bioinformatics/bts635

Dyall, J., Gross, R., Kindrachuk, J., Johnson, R. F., Olinger, G. G., Hensley, L. E., et al. (2017). Middle East respiratory syndrome and severe acute respiratory syndrome: current therapeutic options and potential targets for novel therapies. Drugs 77 (18), 1935-1966. doi:10.1007/s40265-017-0830-1

Geer, L. Y., Marchler-Bauer, A., Geer, R. C., Han, L., He, J., He, S., et al. (2010). The NCBI BioSystems database. Nucleic Acids Res. 38, D492-D496. doi:10.1093/ nar/gkp858 
Giridharan, V. V., Scaini, G., Colpo, G. D., Doifode, T., Pinjari, O. F., Teixeira, A. L., et al. (2020). Clozapine prevents poly (I:C) induced inflammation by modulating NLRP3 pathway in microglial cells. Cells 9 (3), 577. doi:10.3390/ cells 9030577

Hariharan, A., Hakeem, A. R., Radhakrishnan, S., Reddy, M. S., and Rela, M. (2020). The role and therapeutic potential of NF-kappa-B pathway in severe COVID-19 patients. Inflammopharmacol., 1-10. doi:10.1007/s10787-02000773-9

Harrow, J., Frankish, A., Gonzalez, J. M., Tapanari, E., Diekhans, M., Kokocinski, F., et al. 2012). GENCODE: the reference human genome annotation for the ENCODE Project. Genome Res. 22 (9), 1760-1774. doi:10.1101/gr.135350.111

Hirano, T., and Murakami, M. (2020). COVID-19: a new virus, but a familiar receptor and cytokine release syndrome. Immunity 52 (5), 731-733. doi:10. 1016/j.immuni.2020.04.003

Huang, C., Wang, Y., Li, X., Ren, L., Zhao, J., Hu, Y., et al. (2020). Clinical features of patients infected with 2019 novel coronavirus in Wuhan, China. Lancet 395 (10223), 497-506. doi:10.1016/S0140-6736(20)30183-5

Huang, I., Pranata, R., Lim, M. A., Oehadian, A., and Alisjahbana, B. (2020). C-reactive protein, procalcitonin, D-dimer, and ferritin in severe coronavirus disease-2019: a meta-analysis. Ther. Adv. Respir. Dis. 14, 1753466620937175. doi:10.1177/1753466620937175

Josset, L., Menachery, V. D., Gralinski, L. E., Agnihothram, S., Sova, P., Carter, V. S., et al. (2013). Cell host response to infection with novel human coronavirus EMC predicts potential antivirals and important differences with SARS coronavirus. MBio 4 (3), e00165-13. doi:10.1128/mBio.00165-13

Juncal-Ruiz, M., Riesco-Dávila, L., Ortiz-García de la Foz, V., Martínez-Garcia, O., Ramírez-Bonilla, M., Ocejo-Viñals, J. G., et al. (2018). Comparison of the antiinflammatory effect of aripiprazole and risperidone in 75 drug-naïve first episode psychosis individuals: a 3 months randomized study. Schizophr. Res. 202, 226-233. doi:10.1016/j.schres.2018.06.039

Kanehisa, M., Goto, S., Furumichi, M., Tanabe, M., and Hirakawa, M. (2010). KEGG for representation and analysis of molecular networks involving diseases and drugs. Nucleic Acids Res. 38, D355-D360. doi:10.1093/nar/gkp896

Karadsheh Adli, J. (2020). Re: Mahase Elisabeth. Covid-19: What treatments are being 478 investigated? BMJ 368, m1252, 2020 . Available at https://www.bmj. com/content/368/bmj.m1252/rr-1

Kato, T., Monji, A., Hashioka, S., and Kanba, S. (2007). Risperidone significantly inhibits interferon-gamma-induced microglial activation in vitro. Schizophr. Res. 92 (1-3), 108-115. doi:10.1016/j.schres.2007.01.019

Law, C. W., Chen, Y., Shi, W., and Smyth, G. K. (2014). voom: precision weights unlock linear model analysis tools for RNA-seq read counts. Genome Biol. 15 (2), R29. doi:10.1186/gb-2014-15-2-r29

Lee, S. W., Yang, J. M., Moon, S. Y., Yoo, I. K., Ha, E. K., Kim, S. Y., et al. (2020). Association between mental illness and COVID-19 susceptibility and clinical outcomes in South Korea: a nationwide cohort study. Lancet Psychiatry 7 (12), 1025-1031. doi:10.1016/S2215-0366(20)30421-1

Love, M. I., Huber, W., and Anders, S. (2014). Moderated estimation of fold change and dispersion for RNA-seq data with DESeq2. Genome Biol. 15 (12), 550. doi:10.1186/s13059-014-0550-8

Luo, W., and Brouwer, C. (2013). Pathview: an R/Bioconductor package for pathway-based data integration and visualization. Bioinformatics 29 (14), 1830-1831. doi:10.1093/bioinformatics/btt285

Mangalmurti, N., and Hunter, C. A. (2020). Cytokine storms: understanding COVID-19. Immunity 53 (1), 19-25. doi:10.1016/j.immuni.2020.06.017

Manjili, R. H., Zarei, M., Habibi, M., and Manjili, M. H. (2020). COVID-19 as an acute inflammatory disease. J. Immunol. 205 (1), 12-19. doi:10.4049/jimmunol. 2000413

Mayoral Van-Son, J., Gómez-Revuelta, M., Ayesa-Arriola, R., Vázquez-Bourgón, J., Foz, V. O.-G. de. la., Ruiz-Veguilla, M., et al. (2021). Comparison of aripiprazole and risperidone effectiveness in first episode non-affective psychosis: rationale and design of a prospective, randomized, 3-phase, investigator-initiated study (PAFIP-3). Rev. Psiquiatr. Salud Ment. doi:10.1016/j.rpsm.2021.01.004

Mehta, P., McAuley, D. F., Brown, M., Sanchez, E., Tattersall, R. S., and Manson, J. J. (2020). COVID-19: consider cytokine storm syndromes and immunosuppression. Lancet 395 (10229), 1033-1034. doi:10.1016/S01406736(20)30628-0

Metz, M., Staubach, P., Bauer, A., Brehler, R., Gericke, J., Kangas, M., et al. (2017). Clinical efficacy of omalizumab in chronic spontaneous urticaria is associated with a reduction of FceRI-positive cells in the skin. Theranostics 7 (5), 1266-1276. doi:10.7150/thno.18304

Nemani, K., Li, C., Olfson, M., Blessing, E. M., Razavian, N., Chen, J., et al. (2021). Association of psychiatric disorders with mortality among patients with COVID-19. JAMA Psychiatry. doi:10.1001/jamapsychiatry.2020.4442

Obuchowicz, E., Bielecka-Wajdman, A. M., Paul-Samojedny, M., and Nowacka, M. (2017). Different influence of antipsychotics on the balance between pro- and anti-inflammatory cytokines depends on glia activation: an in vitro study. Cytokine 94, 37-44. doi:10.1016/j.cyto.2017.04.004

Pedersen, J., Coskun, M., Soendergaard, C., Salem, M., and Nielsen, O. H. (2014). Inflammatory pathways of importance for management of inflammatory bowel disease. World J. Gastroenterol. 20 (1), 64-77. doi:10.3748/wjg.v20. i1.64

Prieto, C., and Barrios, D. (2020). RaNA-Seq: interactive RNA-Seq analysis from FASTQ files to functional analysis. Bioinformatics 36 (6), 1955-1956. doi:10. 1093/bioinformatics/btz854

Riva, L., Yuan, S., Yin, X., Martin-Sancho, L., Matsunaga, N., Pache, L., et al. (2020). Discovery of SARS-CoV-2 antiviral drugs through large-scale compound repurposing. Nature 586 (7827), 113-119. doi:10.1038/s41586020-2577-1

Rubino, S., Kelvin, N., Bermejo-Martin, J. F., and Kelvin, D. (2020). As COVID-19 cases, deaths and fatality rates surge in Italy, underlying causes require investigation. J. Infect. Dev. Ctries. 14 (3), 265-267. doi:10.3855/jidc.12734

Sánchez-Céspedes, J., Martínez-Aguado, P., Vega-Holm, M., Serna-Gallego, A., Candela, J. I., Marrugal-Lorenzo, J. A., et al. (2016). New 4-Acyl-1phenylaminocarbonyl-2-phenylpiperazine derivatives as potential inhibitors of adenovirus infection. Synthesis, biological evaluation, and structureactivity relationships. J. Med. Chem. 59 (11), 5432-5448. doi:10.1021/acs. jmedchem.6b00300

Smyth, G. K., Michaud, J., and Scott, H. S. (2005). Use of within-array replicate spots for assessing differential expression in microarray experiments. Bioinformatics 21 (9), 2067-2075. doi:10.1093/bioinformatics/bti270

Tay, M. Z., Poh, C. M., Rénia, L., MacAry, P. A., and Ng, L. F. P. (2020). The trinity of COVID-19: immunity, inflammation and intervention. Nat. Rev. Immunol. 20 (6), 363-374. doi:10.1038/s41577-020-0311-8

Vabret, N., Britton, G. J., Gruber, C., Hegde, S., Kim, J., Kuksin, M., et al. (2020). Immunology of COVID-19: current state of the science. Immunity 52 (6), 910-941. doi:10.1016/j.immuni.2020.05.002

van den Berg, D. F., and Te Velde, A. A. (2020). Severe COVID-19: NLRP3 inflammasome dysregulated. Front. Immunol. 11, 1580. doi:10.3389/fimmu. 2020.01580

Wang, Q., Xu, R., and Volkow, N. D. (2021). Increased risk of COVID -19 infection and mortality in people with mental disorders: analysis from electronic health records in the United States. World Psychiatry 20 (1), 124-130. doi:10.1002/ wps. 20806

Wang, Y., Song, F., Zhu, J., Zhang, S., Yang, Y., Chen, T., et al. 2017). GSA: genome sequence archive *. Genomics Proteomics Bioinformatics 15 (1), 14-18. doi:10. 1016/j.gpb.2017.01.001

Weston, S., Coleman, C. M., Haupt, R., Logue, J., Matthews, K., Li, Y., et al. (2020). Broad anti-coronavirus activity of Food and drug administration-approved drugs against SARS-CoV-2 in vitro and SARS-CoV in vivo. J. Virol. 94 (21), e01218-20. doi:10.1128/JVI.01218-20

Wu, C., Chen, X., Cai, Y., Xia, J., Zhou, X., Xu, S., et al. 2020). Risk factors associated with acute respiratory distress syndrome and death in patients with coronavirus disease 2019 pneumonia in Wuhan, China. JAMA Intern. Med. 180 (7), 934-943. doi:10.1001/jamainternmed.2020.0994

Wu, D., and Yang, X. O. (2020). TH17 responses in cytokine storm of COVID-19: an emerging target of JAK2 inhibitor Fedratinib. J. Microbiol. Immunol. Infect. 53 (3), 368-370. doi:10.1016/j.jmii.2020.03.005

Xiong, Y., Liu, Y., Cao, L., Wang, D., Guo, M., Jiang, A., et al. (2020). Transcriptomic characteristics of bronchoalveolar lavage fluid and peripheral blood mononuclear cells in COVID-19 patients. Emerg. Microbes Infect. 9 (1), 761-770. doi:10.1080/22221751.2020.1747363

Yalcin, A. D., Cilli, A., Bisgin, A., Strauss, L. G., and Herth, F. (2013). Omalizumab is effective in treating severe asthma in patients with severe cardiovascular complications and its effects on sCD200, d-dimer, CXCL8, 25-hydroxyvitamin D and IL-1 $\beta$ levels. Expert Opin. Biol. Ther. 13 (9), 1335-1341. doi:10.1517/ 14712598.2013 .819338 
Yalcin, A. D., and Yalcin, A. N. (2021). Future perspective: biologic agents in patients with severe COVID-19. Immunopharmacol. Immunotoxicol. 43 (1), 1-7. doi:10.1080/08923973.2020.1818770

Zeng, Z., Yu, H., Chen, H., Qi, W., Chen, L., Chen, G., et al. (2020). Longitudinal changes of inflammatory parameters and their correlation with disease severity and outcomes in patients with COVID-19 from Wuhan, China. Crit. Care 24 (1), 525. doi:10.1186/s13054-020-03255-0

Zhang, Q., Lenardo, M. J., and Baltimore, D. (2017). 30 Years of NF-kB: a blossoming of relevance to human pathobiology. Cell 168 (1-2), 37-57. doi:10.1016/j.cell.2016.12.012

Zhou, F., Yu, T., Du, R., Fan, G., Liu, Y., Liu, Z., et al. (2020). Clinical course and risk factors for mortality of adult inpatients with COVID-19 in Wuhan, China: a retrospective cohort study. Lancet 395 (10229), 1054-1062. doi:10.1016/ S0140-6736(20)30566-3

Conflict of Interest: BC-F has received unrestricted research funding from Instituto de Salud Carlos III, MINECO, Gobierno de Cantabria, Spanish Network for Research in Mental Health (CIBERSAM), from the seventh European Union Framework Program and Lundbeck. He has also received honoraria for his participation as a consultant and/or as a speaker at educational events from Janssen Johnson \& Johnson, Mylan, Lundbeck, and Otsuka Pharmaceuticals. MR-V has received unrestricted research funding from Instituto de Salud Carlos III. He has also received honoraria for his participation as a consultant and/or as a speaker at educational events from Janssen, Lundbeck, and Otsuka Pharmaceuticals. JV-B has received unrestricted research funding from Instituto de Investigación Marqués de Valdecilla (IDIVAL). He has also received honoraria for his participation as a consultant and/or as a speaker at educational events from Janssen-Cilag and Lundbeck. JC has received honoraria as a speaker from Novartis, Astellas Pharma, Pfizer, MSD Janssen Pharmaceuticals, and AstraZeneca, outside the submitted work. He has also received report grants from Instituto de Salud Carlos III, Spanish Government, co-financed by the European Development Regional Fund "A way to achieve Europe," during the conduct of the study.

The remaining authors declare that the research was conducted in the absence of any commercial or financial relationships that could be construed as a potential conflict of interest.

Copyright (c) 2021 Crespo-Facorro, Ruiz-Veguilla, Vázquez-Bourgon, SánchezHidalgo, Garrido-Torres, Cisneros, Prieto and Sainz. This is an open-access article distributed under the terms of the Creative Commons Attribution License (CC BY). The use, distribution or reproduction in other forums is permitted, provided the original author(s) and the copyright owner(s) are credited and that the original publication in this journal is cited, in accordance with accepted academic practice. No use, distribution or reproduction is permitted which does not comply with these terms. 